\title{
Numerical and experimental study of the multi-axial quasi-static strength of clinched connections
}

\author{
Sam Coppieters • Pascal Lava • Renaat Van Hecke • \\ Steven Cooreman · Hugo Sol • Paul Van Houtte • \\ Dimitri Debruyne
}

Received: 11 November 2011 / Accepted: 9 March 2012 / Published online: 29 March 2012

(C) Springer-Verlag France 2012

\begin{abstract}
Conventionally, the mechanical strength of clinched connections is determined by a single shear lap test and/or a pull-out test. However, in most practical applications a combination of shear and pull-out is exerted on the clinched joint. This paper deals with the development of an Arcan-like device which enables to introduce various shear/tensile ratios in a clinched assembly. An experimental survey of the multi-axial behaviour of a non-cutting single-stroke round clinched connection of two DC05 sheets, which is mild deep drawing steel, is conducted with this modified Arcan setup. These experimental results are used to check the validity of numerical models that predict the strength under multi-axial loading. Since the forming of a clinch is a fairly complex sheet metal operation, a good knowledge of the plastic material properties and the frictional behaviour is of the utmost importance to perform a
\end{abstract}

S. Coppieters $(\varangle) \cdot$ P. Lava $\cdot$ R. Van Hecke $\cdot$ D. Debruyne Department of Mechanical Engineering,

Catholic University College, K.U.Leuven Association,

Gebroeders De Smetstraat 1, 9000 Ghent, Belgium

e-mail: sam.coppieters@kahosl.be

S. Cooreman

OCAS NV, Pres. J.F. Kennedylaan 3, 9060 Zelzate, Belgium

H. Sol

Department of Mechanics of Materials and Constructions, Vrije Universiteit Brussel, Pleinlaan 2, 1050,

Brussel, Belgium

P. Van Houtte $\cdot$ D. Debruyne

Department of Metallurgy and Materials Engineering,

K.U. Leuven, Kasteelpark Arenberg 44 bus 2450,

3001, Heverlee, Belgium sufficiently accurate FEA simulation. The impact of these factors on the multi-axial loading behaviour of the DC05-DC05 connection is investigated.

Keywords Clinching • Sheet metal • Multi-axial loading $\cdot$ Modified Arcan setup

\section{Introduction}

Clinching of sheet metal is a mechanical joining technique which involves a localized cold deformation of the sheets without the use of additional elements such as bolts or rivets as traditional joining methods do. The process is executed with the aid of relatively simple tools such as a punch and a die. Although the types of clinched joints are numerous [1], the clinch process can be categorised into different groups depending on the geometry of the clinching tools. New developments such as dieless clinching [2] and clinch-adhesive joint technology [3, 4] indicate that the clinch technology is rapidly developing. In practice, however, the simple round and rectangular tools are still the most applied tool shapes. This paper deals with a clinched joint produced by a closed round die. Such a joint has some benefits compared to rectangular clinched joints. The latter technique involves the creation of slits in the sheet(s) which act as a stress raiser, and, as a consequence, might lower the fatigue resistance. In addition, the shear strength of a rectangular connection highly depends on the loading direction. As such, the orientation of a rectangular joint is a critical issue when the loading direction is not known a priori. Nevertheless, in some applications (e.g. materials with a limited ductility) the rectangular joint can be more favourable. It 
must be noted that the presented Arcan-like device is not limited to testing of axisymmetric clinched joints. The cross section of a round axisymmetric joint is shown in Fig. 1. The right panel shows a picture of an encapsulated cross-section which is properly polished to reveal the interface between the assembled sheets. The left panel shows the contour of the joint measured by an optical microscope. The mechanical behaviour of these type of connections is solely dictated by the final geometry. As such, the influence of the geometry of the clinching tools with respect to the final geometry has been investigated with the aid of finite element techniques in the past [5-7]. The broader goal of such studies on the metal flow during clinching is the optimization of the joint (and tools), e.g. to maximize its strength for a certain application. Oudjene and his coworkers [8] have illustrated this idea by developing a strategy to optimize a clinched joint with respect to the axisymmetrical pull-out strength. Traditionally, the assessment of the strength of clinched connections is based on two basic loading cases: a single shear lap test [9] and a pull-out test [10] and it is generally recognized that the axial strength of clinched connections is much lower compared to the shear strength. Although the single shear lap test and the pull-out test allow the assessment of the mechanical behaviour in a consistent manner, in practice the joint is usually exposed to a combination of shear and pull-out components. It is clear that for a dedicated application the optimization of a clinched joint requires a more complex test allowing to exert random shear/tensile ratios on the joint. In theory, this issue can be solved by using a virtual platform such as the finite element method. However, these results can only be useful if they are able to reproduce the experimentally observed strengths. This paper presents a destructive test method which enables to exert quasi-static multi-axial loading conditions on a clinched connection. In addition, the capability of finite element techniques to reproduce these experimental tests is investigated. Although the mechanisms are completely different, many parallels can be drawn between research on the mechanical behaviour of spot welds, rivets and clinched connections. Langrand and his co-workers have addressed the mechanical behaviour of spot welds [11] and rivets [12] under multi-axial loading. Similar techniques can be developed for testing clinched joints under multi-axial loading. This would allow to investigate crack propagation and failure criteria for clinched joints under multi-axial loading. In addition, such a test would allow to develop simplified joint models. Indeed, since a clinched joint is rather complex in terms of geometry and material state after forming, it would be unrealistic to model thousands of clinched connections in a structure because of the exuberant computational cost. Therefore, it is generally accepted to replace the complex model by an equivalent, computationally efficient model. The focus in this paper is on the mechanical behaviour of a clinched joint which connects two DC05 sheets. The next section presents a modified Arcan setup which allows to experimentally investigate a clinched joint under varying shear/tensile ratios. Section "Simulation of the clinch forming process" embarks on the forming process of this type of connection and presents a strategy to perform finite element simulations of this forming operation. In Section "Reproducing the multi-axial loading strength using FEA" the numerical models to predict the strength under multi-axial loading are validated with the aid of the experimental results. Here it is also shown that a comparison between numerical and experimental results obtained by multi-axial loading offers an excellent opportunity to assess the identified
Fig. 1 Single-stroke non-cutting round clinched joint (DC05-DC05). Right panel: encapsulated cross-section with interlock F. Left panel: measurement by an optical microscope

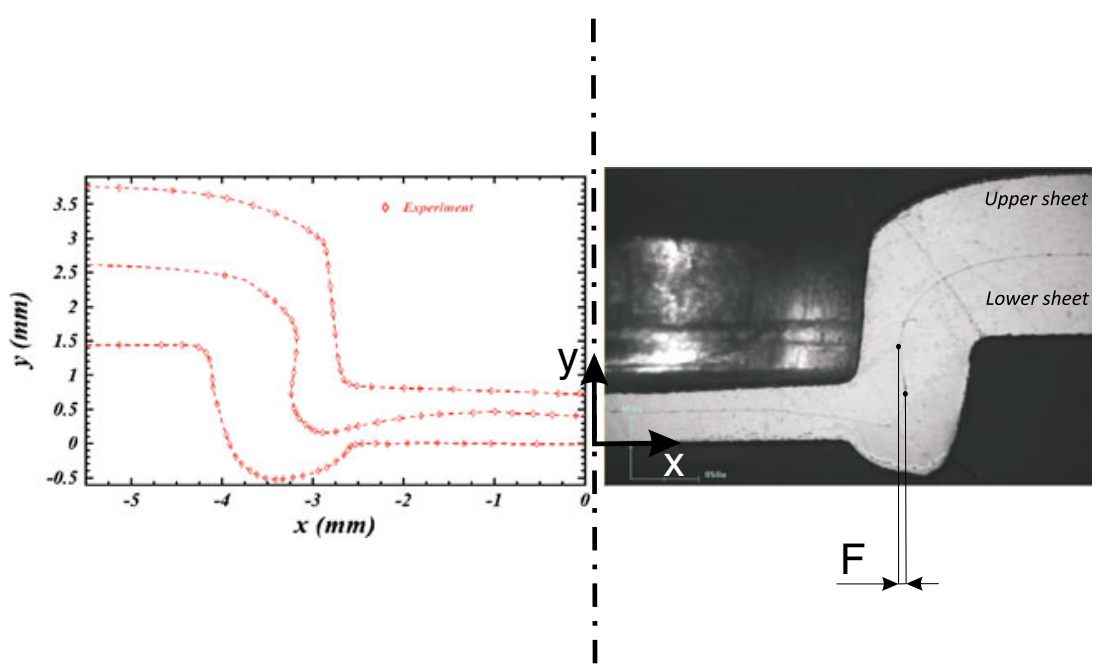


material properties of the sheet metal used in this study. We conclude in Section "Conclusion and future work".

\section{The modified Arcan setup}

Several experimental procedures have been developed to enable mixed-mode testing on a specimen. One of these procedures is the so-called Arcan fixture which was originally developed to characterize composite materials [13]. The valuable benefit of this test is that it can be conducted on a standard tensile bench or any other single-axis device. This test procedure has been used in a variety of engineering domains of which the majority of the applications can probably be found in fracture mechanics [14]. Since the essence of this experimental set up is to simultaneously apply and control tensile and shear loads on a specimen, it has also drawn the attention of researchers in the field of joint design. A modification of the Arcan fixtures allows to investigate for example the behaviour of spot welds [15] or riveted joints $[16,17]$ under multi-axial loads. In this section a modified Arcan fixture which enables the mixed-mode loading of clinched connections is presented and details of the specimen design are given. Many variations on the Arcan fixture can be found in literature and the design of the modified Arcan setup depends on the design of the specimen to be tested. In the case of destructive joint testing, the fixtures of the Arcan test have to be able to clamp an assembly with sufficient clamping force. Figure 2 shows the basics of the experimental set up. The device consists of two separate disk halves which enable clamping of a specimen. Once the device is provided with a specimen, the two disk halves are connected via this assembled specimen. The next step is then to mount it into a tensile machine: one of the disk halves is connected to a moving cross-head while the other is fixed. In general there are two methods to do so [18]. The first method uses a connection which allows the rotation of the disk halves during testing. The second method prevents this rotation by using a fixed connection. The design shown in Fig. 2 allows both methods by using a multiple-pin clevis. If only pin 1 and pin 2 are inserted into the clevis, then rotation is allowed and any side load on the load cell is avoided. The "fixed" mounting can be achieved by adding pin 3 to the configuration of method 1 . Unlike the first method, the second can exert a potentially damaging side load on the load cell, and, as consequence, adequate precautions must be taken when applying this method. Rotation of the complete system by replacing the clevis position on the disk halves changes the tensile/shear ratio. The current design shown in Fig. 2

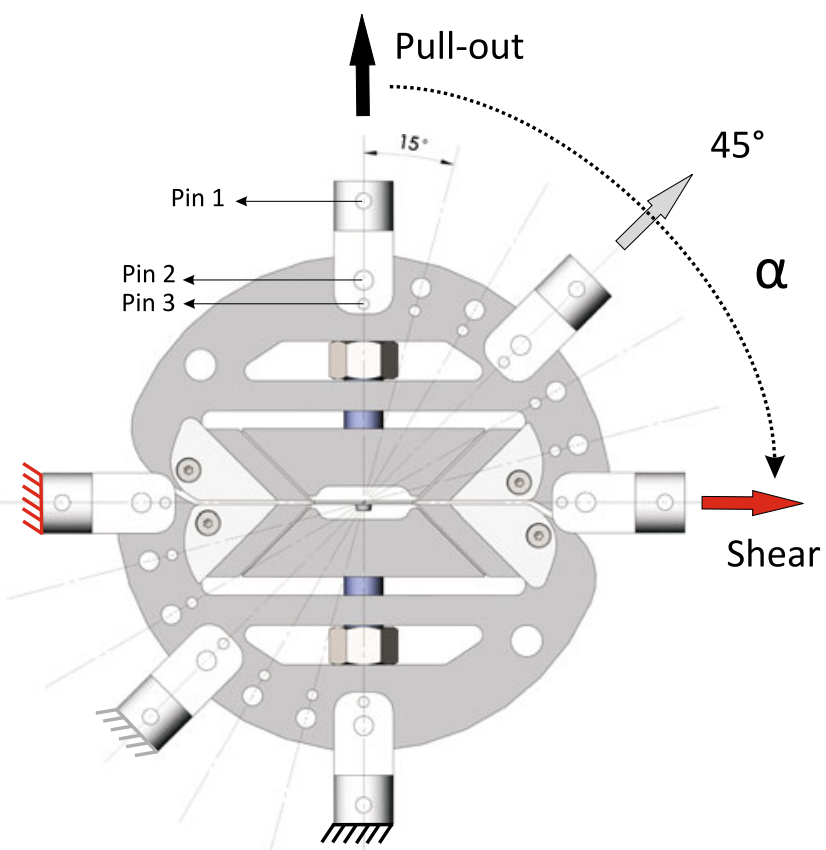

Fig. 2 Basics of the modified Arcan set up for clinched connections. Three loading directions are shown: pull-out $\left(\alpha=0^{\circ}\right)$, $\alpha=45^{\circ}$ and shear loading $\left(\alpha=90^{\circ}\right)$

allows for the following angular positions: $(\alpha=0,15$, $\left.30,45,60,75,90^{\circ}\right)$ of which pull-out $\left(\alpha=0^{\circ}\right), \alpha=45^{\circ}$ and shear loading $\left(\alpha=90^{\circ}\right)$ are depicted in the figure. Figure 3 shows a partly exploded view of the modified Arcan test used in this study. The Arcan specimen is

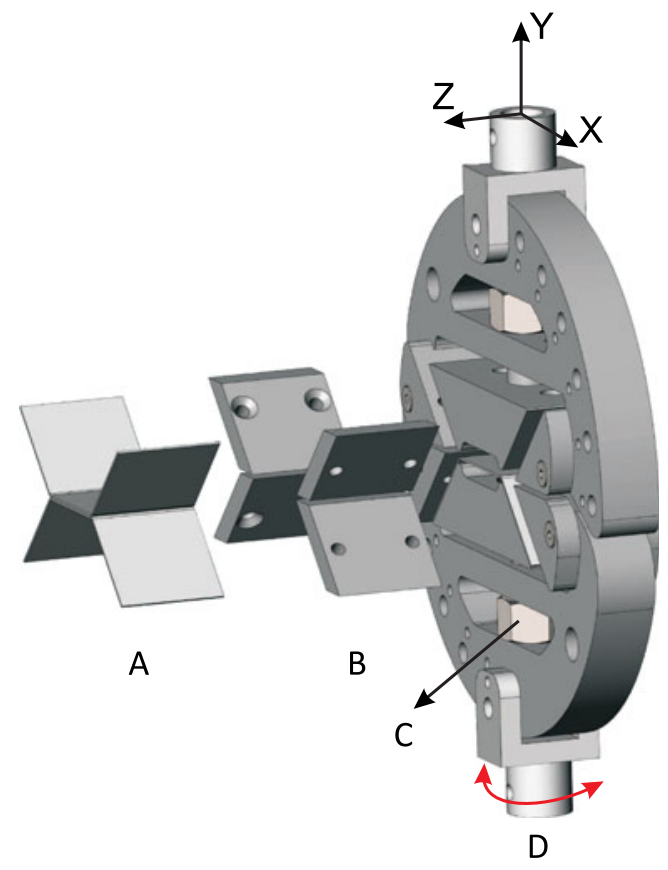

Fig. 3 Partly exploded view of the modified Arcan setup. $A$ The Arcan specimen $B$ back plates $C$ tightening nut $D$ Clevis 

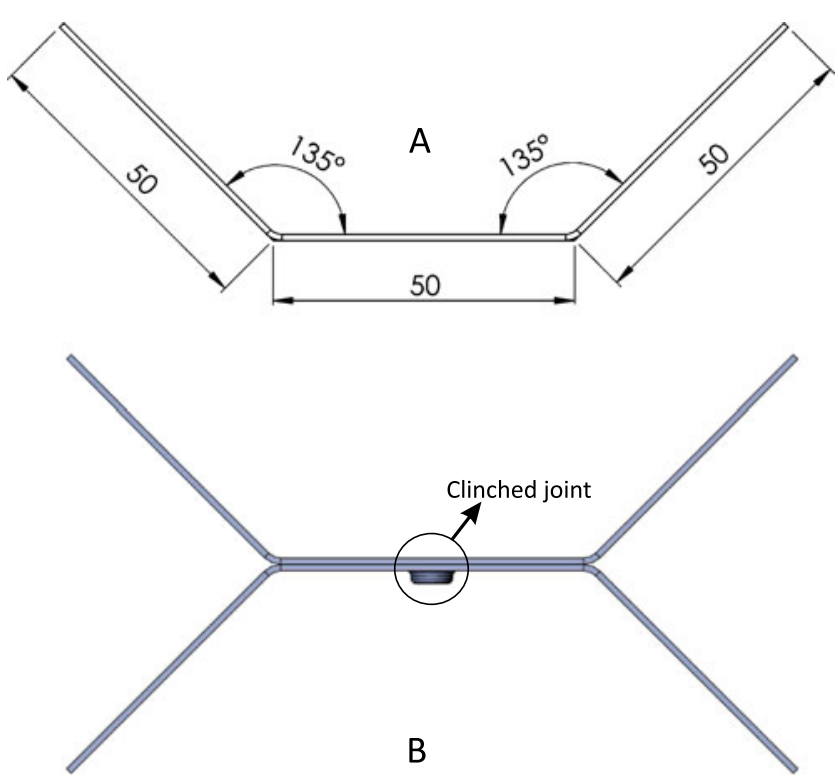

Fig. 4 The Arcan specimen for clinched connections. A A folded strip with a width of $50 \mathrm{~mm} B$ the assembled Arcan specimen

denoted $A$ in this figure and more details concerning its dimensions can be found in Fig. 4. The current design requires two strips of $150 \times 50 \times t \mathrm{~mm}$, with $\mathrm{t}$ the sheet thickness, which need to be folded as shown in the upper part of Fig. 4. The two folded strips must be assembled as shown in the lower part of the same figure. In order to be able to sufficiently clamp specimens with varying sheet thicknesses $t$, the clamping unit consists of serrated back plates (denoted B in Fig. 3) which can be easily changed. The clamping is enforced by tightening the nuts shown in Fig. 3. Figure 5 shows the modified Arcan device mounted with an angular position $\alpha=15^{\circ}$ into a regular tensile bench with a capacity of $10 \mathrm{kN}$. The data readily available from such

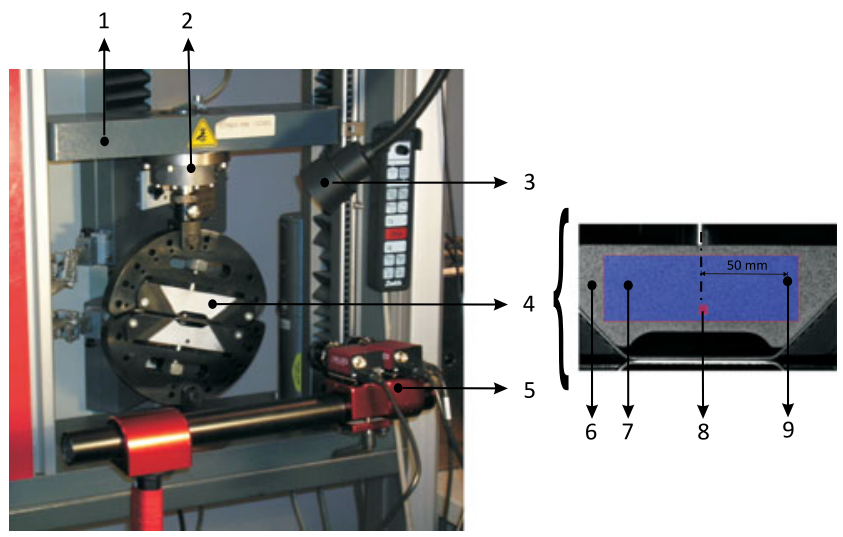

Fig. 5 The modified Arcan set up mounted in a standard tensile bench. 1 Cross-head 2 load cell 3 lamp 4 upper half disk 5 camera's 6 speckle pattern 7 area of interest 8 subset 9 point at $50 \mathrm{~mm}$ from the centre an experiment on a tensile bench is the load-elongation curve. The force during separation can be measured by the load cell, denoted 2 in Fig. 5, and the cross-head (denoted 1) delivers the vertical displacement along the axis of the actuator. However, if method 1 is adopted, each disk halve can rotate in its clevis and this behaviour cannot be measured with a classical extensometer. Therefore, the setup shown in Fig. 5 is augmented with an optical-numerical measuring technique called Digital Image Correlation (DIC). The Charged-Coupled Device (CCD) cameras (denoted 5, in Fig. 5) take synchronized images of the Arcan fixtures in the reference and operating state of the Arcan device. Next, these images are computationally compared in our in-house DIC platform MatchID (www.matchID.org). From this the displacement fields at the surface of the Arcan fixtures are retrieved for different loading steps. The DIC algorithm used in this study is a so-called subsetbased method which correlates the movement of a pixel and its neighbourhood from the initial image in consecutive images. A prerequisite to enable the discrimination between different groups of pixels, which is called a subset, is to obtain an image with a distinctive pattern. Therefore, as shown in Fig. 5, a random speckle pattern is sprayed on the surface of the Arcan fixtures. Since in many cases the ambient light is not enough to obtain a bright image without using unacceptable large exposures times or aperture settings, supplemental light (denoted 3 ) is added.

Figure 6 shows the experimental data which can be acquired during the modified arcan test. The results shown here are from a clinched connection in the angular position $\alpha=0^{\circ}$ and the frame in which the results are presented is indicated in Fig. 3. The upper panel shows the comparison between the displacement $V$ in the $Y$-direction measured by the cross-head and DIC. As can be inferred, these measurements are in good agreement. The middle panel shows the displacement $\mathrm{W}$ in the $Z$-direction of a point lying $50 \mathrm{~mm}$ from the centre of the joint, see Fig. 5. The lower panel shows the average rotation $\phi$ of a disk half in the $X Y$-plane, see Fig. 3.

Both $\mathrm{W}$ and $\phi$ show a steep drop in the beginning of the test. This is due to the mechanical tolerances in the system and the joint. However, this can be overcome by applying a preload of approximately $50 \mathrm{~N}$. As indicated by the black dotted line, from this point on, stable results can be expected. It can be inferred that the maximum displacement $\mathrm{W}$ from this point on is approximately $0.05 \mathrm{~mm}$. The maximum average rotation $\phi$ in the test is about $0.002 \mathrm{rad}$ which confirms that in this case (method $1, \alpha=0^{\circ}$ ) the setup behaves like a true pull-out test. 


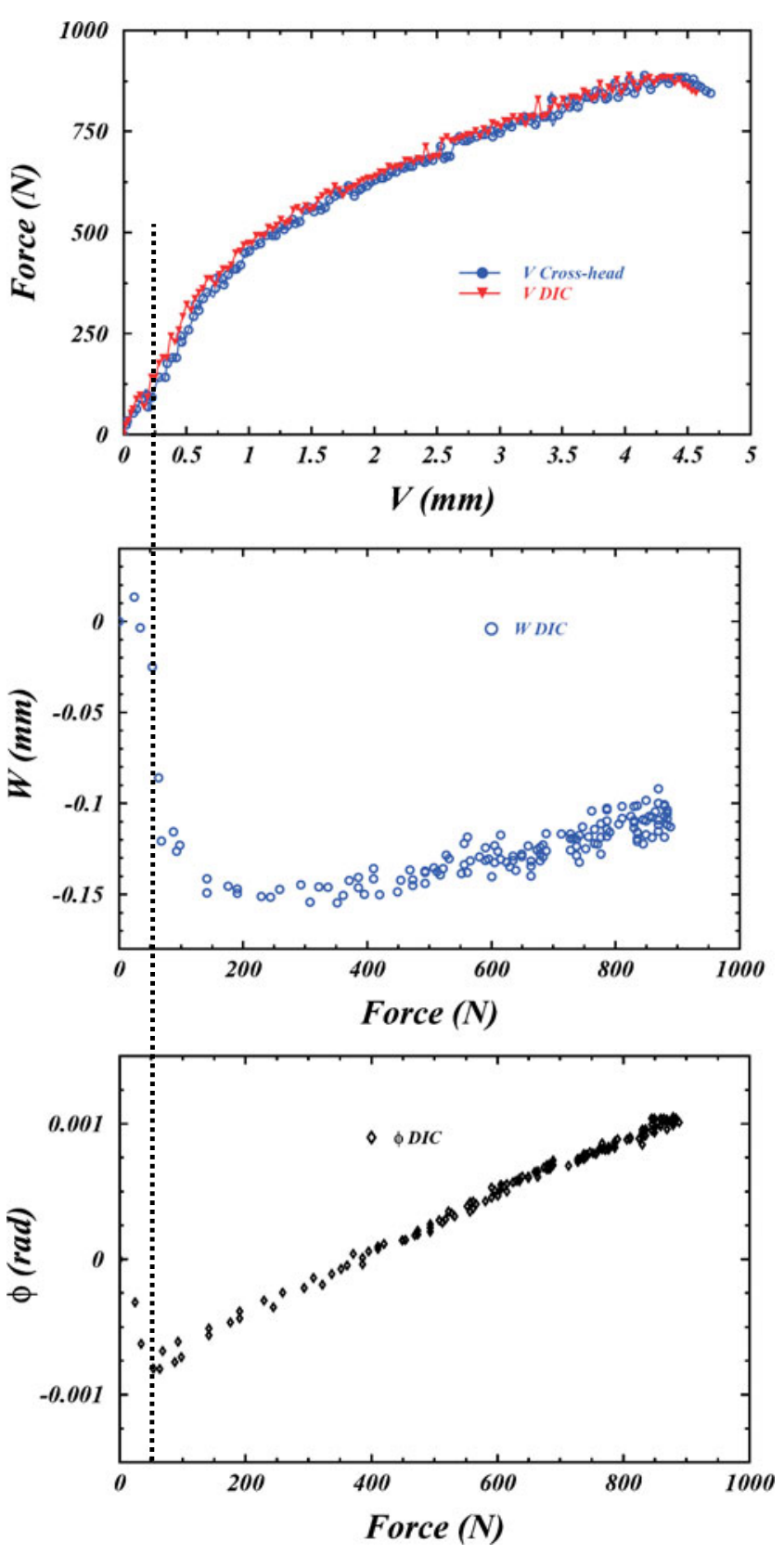

Fig. 6 Acquired data during a modified Arcan test. Results from an angular position $\alpha=0^{\circ}$. Upper panel: Comparison between cross-head measurement and DIC. Middle panel: Out-off plane displacement in function of the force. Lower panel: Average (inplane) rotation in the AOI in function of the force

\section{Simulation of the clinch forming process}

Mechanics of the clinch forming process

Figure 7 shows an experimental process graph (forcestroke curve) measured during clinch forming. The process graph of such a process can be used to distinguish several stages during the forming. Up until

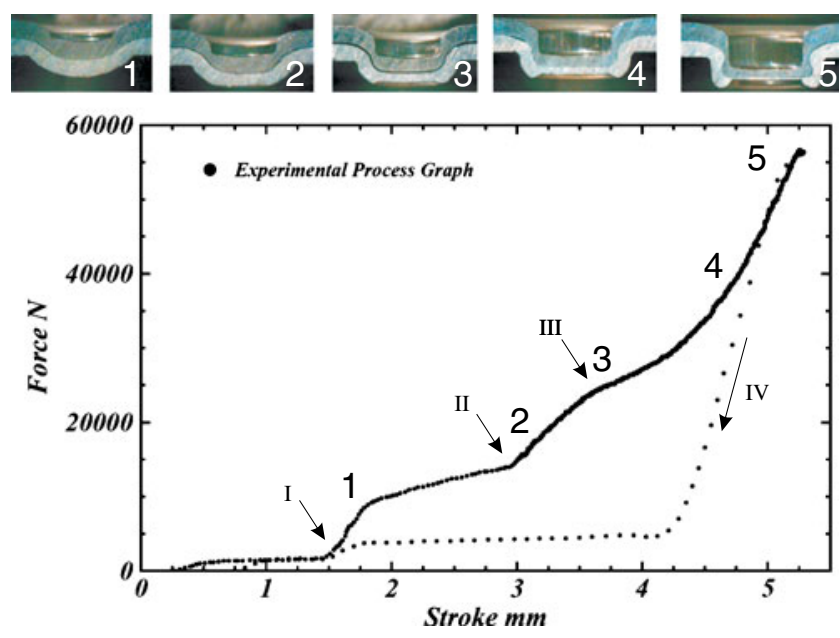

Fig. 7 An experimental process graph of the clinch forming process and the associated deformation modes

point $I$, the auxiliary tools (which can comprise a blank holder and/or a punch stripper) are exerting a certain force on the blanks. The sudden increase at point I indicates the initial insertion of the sheet material into the die. Between points I and II, the upper part of the die is filled and the material touches the anvil of the die which causes a steep increase of the force. From point III up until the end of the process the material is forced to fill the ring groove in the die while the interlock is created. Figure 7 also shows different deformation modes (cross section of the joint) of the joint during the forming.

The latter mechanism - the creation of F-is the key feature of the joining method. Typically the thickness at the base of the joint undergoes a huge reduction and also other regions of the joint are subjected to severe plastic deformation. Since the order of these strains is far beyond the maximum uniform strain of the sheet, the post-necking hardening behaviour needs to be identified in order to perform a finite element simulation of the forming process. In addition, the frictional conditions (sheet-sheet contact and tool-sheet contact) in the forming simulation need to be identified. The next section deals with these identification problems.

Identification of strain hardening and friction

\section{Strain hardening}

The clinching process described in the previous section inherently involves severe plastic deformation of the sheet metal. It is well known that in such a case a good knowledge of the material behaviour is of utmost importance to perform a sufficiently accurate simulation. 
Since most FE codes use phenomenological models to describe the concept of a yield criterion and hardening behaviour, the parameters in these models have to be identified. Conventionally this is done by conducting standard experiments such as a tensile test, a compression test, torsion test or a shear test. The tensile test is probably the most widely adopted material test but has serious limitations when the behaviour at large plastic strains has to be identified. This is usually countered by extrapolation of the pre-necking hardening behaviour using a predefined hardening law, in the remainder of this work referred to as Extrapolation method $(E M)$. Unfortunately, such a procedure may give very different results depending on the hardening law fitted to the experimental pre-necking data.

Clinching is often the last step in the production process, and, as a result, it are often semi-finished products which need to be clinched. The local material properties of these semi-finished products are usually not known. Since it has been shown in $[7,10]$ that ignoring the material state leads to an unrealistic prediction, there are only two options here. The first requires the simulation of the preceding forming operations to take the deformation history into account. Unfortunately, the accuracy of this is usually hampered by the limited information regarding the initial material properties and process-specific conditions. The second method is to identify the local material properties by experimental testing. This can be done by locally removing material which is then subjected to a material test. To cope with the limitations of the tensile test two alternative material tests are used, namely a Multi-Layered Upsetting Test (MLUT) and the Post-Necking Tensile Experiment (PNTE). Compression of stacked sheets has been investigated in $[19,20]$. The identification of strain hardening behaviour beyond necking in a tensile has been targeted in [21, 22]. Details on the methods used in this paper can be found in [23, 24].

\section{Frictional behaviour}

Most sheet metal forming applications deal with relatively low interface pressures and large sliding lengths between tools and sheet. For these situations sheet metal friction tests are available [25]. In the case of clinching, however, completely different friction conditions prevail. The clinching tools typically exert very high pressures on the sheets while sliding lengths generally remain small. Due to the small dimensions of the clinching tools, the local deformation of the sheets during clinch forming must be regarded as a bulk forming problem. As such, one has to resort to other friction identification methods. The most common method used to determine friction for a forging process is the ring compression test. Since this test uses a ring with typical dimensions (outer diameter:inner diameter:thickness $)=(6: 3: 1)$, the dimensions of the ring would become very small in the case of thin sheets. Alternative methods are often based on the correlation of accurate measurements with numerical responses. Here, the friction condition is iteratively tuned by minimizing the discrepancy between numerical and experimental responses such as shapes or forming loads. Provided that the elasto-plastic material behaviour is known, the inverse procedure yields then a friction condition for which the accuracy depends on the sensitivity of the measured quantities with respect to the unknown parameters. In this section, the strategy to identify the friction conditions in clinch forming of similar materials with equal thicknesses is presented. In this case two friction conditions can be distinguished. There is the tool-sheet contact $\left(\mu_{t}\right)$ and the sheet-sheet interface contact $\left(\mu_{i}\right)$. For both friction conditions the Coulomb friction model is adopted. In addition, because of the high contact pressure between the sheets, the model for the sheet-sheet interface is extended with a shear stress limit $\tau_{\max }$. In other words, subsurface shearing between adjacent sheets occurs when $\tau_{\max }$ is exceeded. The shear stress limit used in this model equals:

$\tau_{\max }=\frac{\sigma_{y}}{\sqrt{3}}$

where $\sigma_{y}$ is the initial von Mises yield stress of the sheet metal.

As a result, the inverse problem under consideration has only two unknowns: the tool-sheet contact $\left(\mu_{t}\right)$ and the sheet-sheet interface $\left(\mu_{i}\right)$. These parameters will be tuned by the minimization of a cost function which expresses the discrepancy between computed and measured responses. The available experimental data are in this case the process graph (see Fig. 7) and the final geometry of the joint (see Fig. 1). Apart from the influences of $\mu_{t}$ and $\mu_{i}$ on the process graph, these parameters will also influence the metal flow which determines the interlock $F$. This parameter represents the amount of material which "hooks" behind the lower sheet. To gain more insight into the relation between $\mu_{t}, \mu_{i}$ and $\mathrm{F}$, numerical simulations were performed in [26] to investigate this. The results of this work are shown in Fig. 8.

Figure 8a clearly shows that $F$ is independent of $\mu_{t}$, regardless of the magnitude of $\mu_{i}$. It can also be inferred from the same panel that $\mu_{i}$ dictates the amount of interlock. In the situation under consideration, the interlock increases with an increasing $\mu_{i}$. This phenomenon is also observed by Jomaa [27] and it 


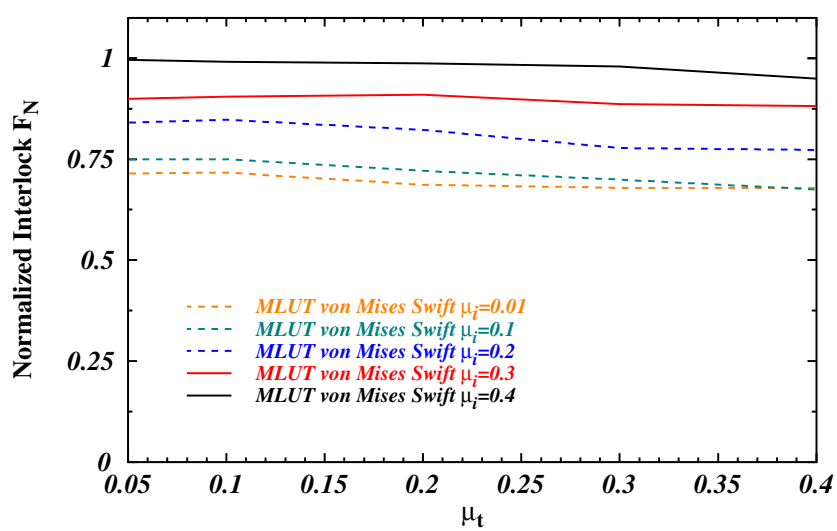

(a)

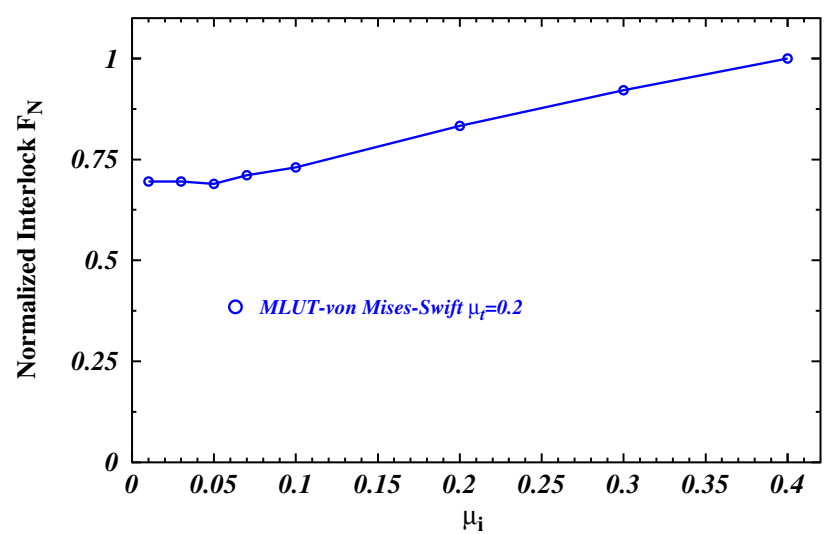

(b)

Fig. 8 a Normalized interlock $F_{N}$ as a function of $\mu_{i}$ and $\mu_{t}$ using a MLUT and a swift law. b Normalized interlock $F_{N}$ as a function of $\mu_{i}$ and $\mu_{t}=0.2$ using a MLUT and a Swift law

can probably be explained by the following. The ring groove in the die allows the lower sheet material to be expelled outwards. This causes the material flow to accelerate towards the ring groove as shown by Mucha [28]. A high interface friction allows the upper sheet to follow the lower sheet resulting in a higher interlock. Figure $8 \mathrm{~b}$ illustrates this behaviour for one particular case where $\mu_{t}=0.2$. This observation allows the inverse identification of $\mu_{t}$ and $\mu_{i}$ to be dealt with separately, though in a specific order:

(1) Determine the frictional condition between the adjacent sheets by minimizing the cost function $C\left(\mu_{i}^{k}\right)$ (Eq. 2). The inverse procedure is "fed" by the experimentally measured interlock $F$. Since it is shown that $\mu_{t}$ has no significant influence on $\mathrm{F}$, this first inverse optimization is done by using an arbitrary (but realistic) value for $\mu_{t}$.

$C\left(\mu_{i}^{k}\right)=\left|F^{\mathrm{num}}\left(\mu_{i}^{k}\right)-F^{\exp }\right|$

where $F^{\text {num }}$ and $F^{\text {exp }}$ are the numerically computed and experimentally measured interlock, re- spectively. $\mu_{i}^{k}$ is the Coulomb friction coefficient between adjacent sheets and the index $k$ refers to the data set (see Table 2) used in the optimization.

(2) Determine the frictional condition between the tools and the sheets by minimizing the cost function $C\left(\mu_{t}^{k}\right)$ (Eq. 3). The inverse procedure is "fed" by the experimentally measured process graph. The $\mu_{i}^{k}$ remains fixed in this step and is equal to the optimized value from step 1.

$C\left(\mu_{t}^{k}\right)=\frac{1}{2} \sum_{i=0}^{N}\left[P_{i}^{\text {exp }}-P_{i}^{\text {num }}\left(\mu_{t}^{k}\right)\right]^{2}$

where $P_{i}^{\exp }$ and $P_{i}^{\text {num }}\left(\mu_{t}^{k}\right)$ are the experimentally measured and numerically computed forces at different positions $i$ of the punch, respectively. $\mu_{t}^{k}$ is the Coulomb friction coefficient between the sheets and the tools, with index $k$ referring to the material set used. $\mathrm{N}$ represents the number of measurements.

It must be noted that this method always requires experimental data. This can be seen as a drawback if the method is used to support the design of new clinching tools. However, new applications can be usually joined with existing tools. The problem is that this give not the optimal solution in terms of mechanical behaviour or joint defects such as cracks. These results obtained with existing tools can be used to tune friction conditions which are then used to optimize tools.

\section{Results}

Since a DC05-DC05 clinched connection is the subject of this paper, the post-necking hardening behaviour of this sheet metal is investigated by the EM, a MLUT and a PNTE. Next, for each identified set of material parameters, the friction conditions are inversely calculated by the strategy presented in Section "Frictional behaviour". This process is shown as a three-step flowchart in Fig. 9 yielding a data set which consists of material and frictional behaviour. The plastic material behaviour is described by:

(1) a yield criterion. In this paper, for the EM and the PNTE the yield surface is described by Hill's 1948 yield criterion. In the case of plane stress conditions, Hill's criterion takes the following form according to the principal axes of anisotropy 1 and 2:

$$
F \sigma_{22}^{2}+G \sigma_{11}^{2}+H\left(\sigma_{11}-\sigma_{22}\right)^{2}+2 N \sigma_{12}^{2}-\sigma_{e q}^{2}=0
$$




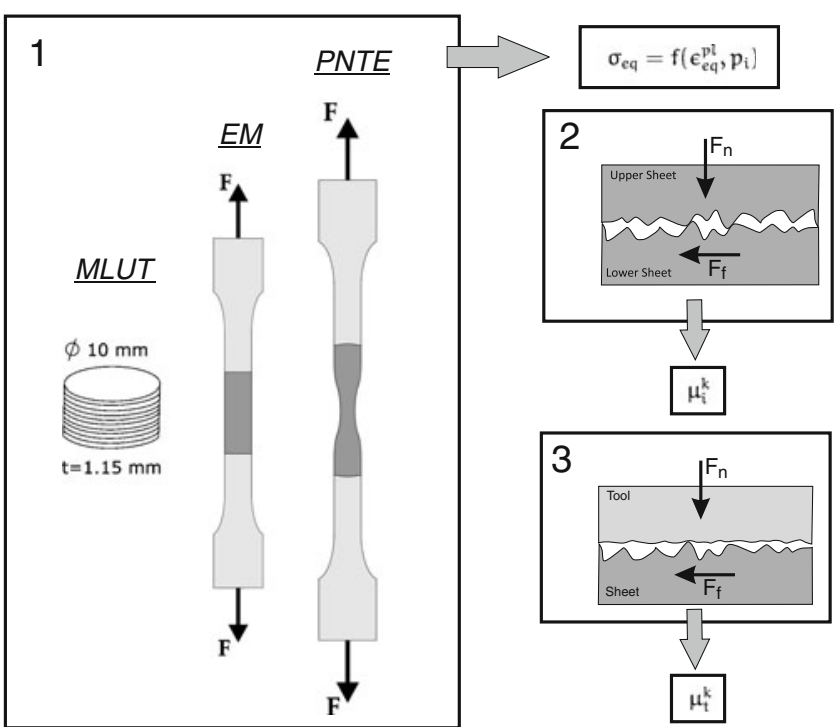

Fig. 9 Three-step identification flow chart. Step 1: Identification of the post-necking hardening behaviour. Step 2: Identification of the friction between the sheets. Step 3: Identification of the friction between sheets and tools

where $F, G, H$ and $N$ are material constants characterizing the state of anisotropic yield behaviour. These parameters are determined in advance based on the Lankford ratio's measured along the rolling direction, the transverse direction and the $45^{\circ}$ direction. Their values can be found in Table 1. For the MLUT and also the EM the von Mises yield criterion is adopted and in the principal stress space it reads as:

$\sigma_{e q}^{2}=\frac{1}{2}\left[\left(\sigma_{1}-\sigma_{2}\right)^{2}+\left(\sigma_{1}-\sigma_{3}\right)^{2}+\left(\sigma_{2}-\sigma_{3}\right)^{2}\right]$

(2) the associated flow rule which is used to express the relationship between the stress state and the plastic strain rate:

$D_{i j}^{p l}=\dot{\lambda} \frac{\partial f\left(\sigma_{i j}\right)}{\partial \sigma_{i j}}$

with $D_{i j}^{p l}$ the plastic part of the rate of deformation tensor, $\dot{\lambda}$ the plastic multiplier and $f\left(\sigma_{i j}\right)$ the yield surface.

(3) a hardening law which describes the evolution of the initial yield surface due to plastic deformation.

Table 1 A priori determined parameters of Hill's 1948 yield criterion

\begin{tabular}{llll}
\hline$F$ & $G$ & $H$ & $N$ \\
\hline 0.2748 & 0.3662 & 0.6338 & 1.523 \\
\hline
\end{tabular}

The post-necking hardening behaviour has to be parameterized, so as to obtain a finite set of unknown parameters $p_{i}$ to be optimized:

$\sigma_{e q}=f\left(\epsilon_{e q}^{p l}, p_{i}\right)$

Two commonly used hardening laws are selected in this study, namely a Swift (Eq. 8) and a Voce law (Eq. 9):

$$
\begin{aligned}
& \sigma_{e q}=K\left(\epsilon_{0}+\epsilon_{e q}^{p l}\right)^{n} \\
& \sigma_{e q}=C\left(1-m e^{-k \epsilon_{e q}^{p l}}\right)
\end{aligned}
$$

First the post-necking hardening behaviour is identified through the three material tests. The results are shown in Fig. 10 and the identified parameters are summarized in Table 2. The experimental interlock $\mathrm{F}^{\exp }$ is measured on a sectioned joint with an optical microscope and an average value of $F^{\exp }=0.068 \mathrm{~mm}$ is found. This value is located in the plateau shown in the right panel of Fig. 8. Step 2 in Fig. 9 resulted in a value $\mu_{i}^{k}<0.1$ for $k=(1,2,3,4,5,6,8)$. As a result, step 2 of the three-step flowchart is performed with a fixed friction condition between adjacent sheets, $\mu_{i}^{k}=$ 0.1 . Figure 11 shows the results of the optimization of $\mu_{t}^{k}$ on the basis of the process graph. Figure 11a shows the force-displacement predictions before optimization. Figure $11 \mathrm{~b}$ clearly shows the convergence of the curves after optimization. The optimized value $\mu_{t}^{k}$ for each material data set can be found in Table 2 . It is clear that the identified friction values shown in Table 2 compensate for the uncertainty regarding the material behaviour at high plastic strains. Indeed, it can be seen from Table 2 that the Voce laws, which exhibit less strain hardening than the Swift laws, are compensated by higher coefficients of friction. In this regard, it must also be noted that the Coulomb friction model is probably not sufficient to describe the complicated friction regimes in clinch forming. In addition, the presented strategy inherently delivers an averaged constant friction value which probably varies during actual forming. All these factors make it difficult to have a physical basis for the assessment of the friction values.

The data sets of Table 2 can in turn be used to simulate the joint forming. A geometrical comparison between the calculated and the experimentally measured final geometry was made in [26]. It was concluded that the alternative identification methods are an improvement over the classical EM, at least in the sense that the results from the alternative tests were more consistent and less dependent on the chosen hardening 
Table 2 Identified material parameter sets for DC05

\begin{tabular}{cllllll}
\hline Hardening law & Method & & & & & \\
\hline Swift & Parameter & $\mathrm{K}(\mathrm{MPa})$ & $n$ & $\epsilon_{0}$ & Yield criterion & $\mu_{t}^{k}$ \\
$k=1$ & EM & 528 & 0.286 & $5.262 \mathrm{E}-03$ & von Mises & 0.167 \\
$k=3$ & EM & 507 & 0.28 & $4.82 \mathrm{E}-03$ & Hill48 & 0.104 \\
$k=5$ & MLUT & 517 & 0.252 & $5.65 \mathrm{E}-04$ & von Mises & 0.176 \\
$k=7$ & PNTE & 467 & 0.23 & $1.00 \mathrm{E}-06$ & Hill48 & 0.137 \\
Voce & Parameter & $\mathrm{C}(\mathrm{MPa})$ & $m$ & $k$ & Yield criterion & $\mu_{t}^{k}$ \\
$k=2$ & EM & 365 & 0.625 & 9.99 & von Mises & 0.809 \\
$k=4$ & EM & 347.69 & 0.628 & 10.75 & Hill48 & 0.568 \\
$k=6$ & MLUT & 422 & 0.685 & 8.01 & von Mises & 0.328 \\
$k=8$ & PNTE & 387.60 & 0.616 & 6.63 & Hill48 & 0.306 \\
\hline
\end{tabular}

law. However, this statement was solely based on the assessment of the final geometry. Further on in this contribution we will simultaneously assess the quality of the identified behaviour through the mechanical behaviour under multi-axial loading.

\section{Reproducing the multi-axial loading strength using FEA}

FE model

From here it is assumed that the results of the FE simulation of the forming process are available. The clinched joint (with the associated material state after forming) is then assembled into an Arcan specimen to simulate the mechanical behaviour under multi-axial loading. Figure 12 shows how the numerical model of the modified Arcan test is built. It is assumed that the Arcan fixtures are infinitely rigid and in the model they are replaced by kinematic couplings, denoted $\mathrm{KC}$

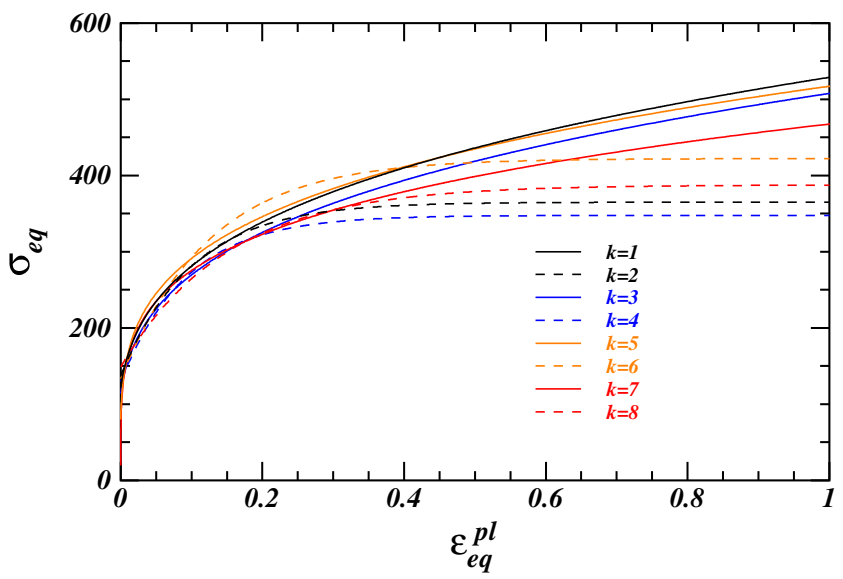

Fig. 10 Identified flow curves for DC05 in Fig. 12. The coupling connects the reference points RP1 and RP2, which are located at the centre of PIN2 from Fig. 2, and the Arcan specimen. The upper Arcan fixture (RP1) is constrained to a specified translation with the magnitude $U$ while rotation around the $Z$ axis is transmitted. The translational degrees of freedom of the lower Arcan fixture are constrained in all

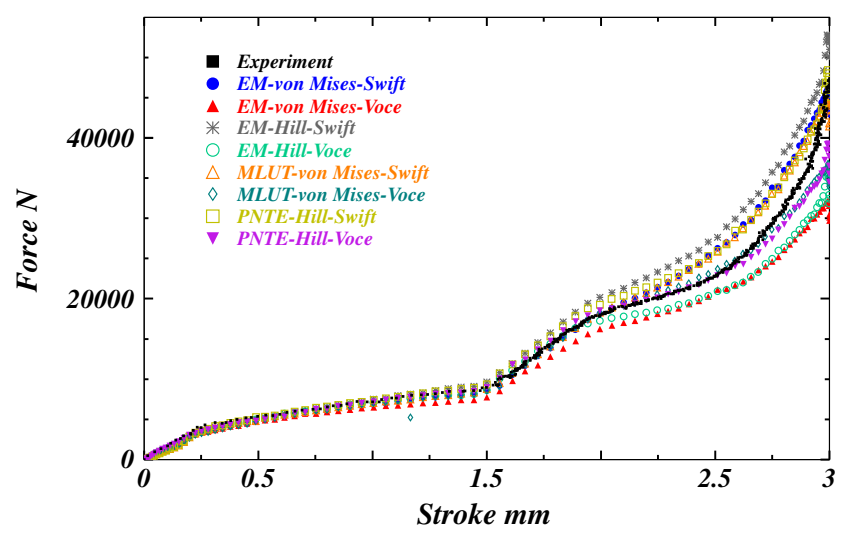

(a)

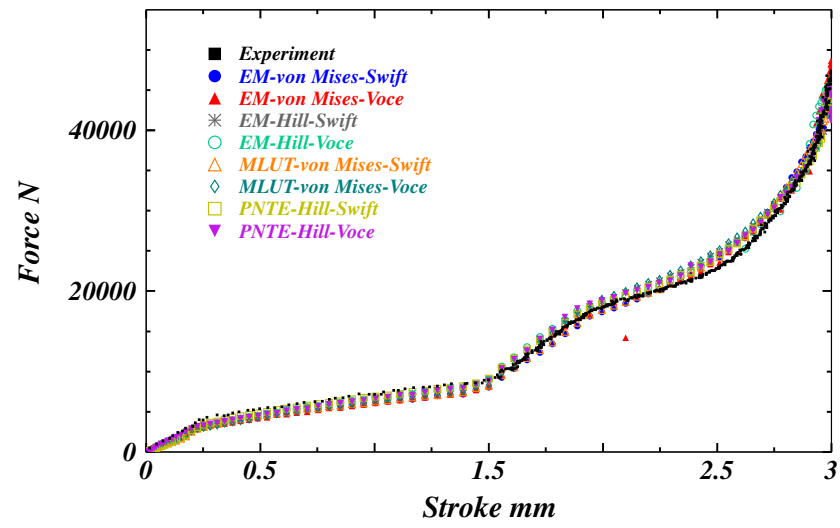

(b)

Fig. 11 a Process graph before optimization $\left(\tau_{\max }=72 \mathrm{MPa}\right.$, $\mu_{i}=0.1$ and $\left.\mu_{t}=0.2\right)$. b process graph after optimization 


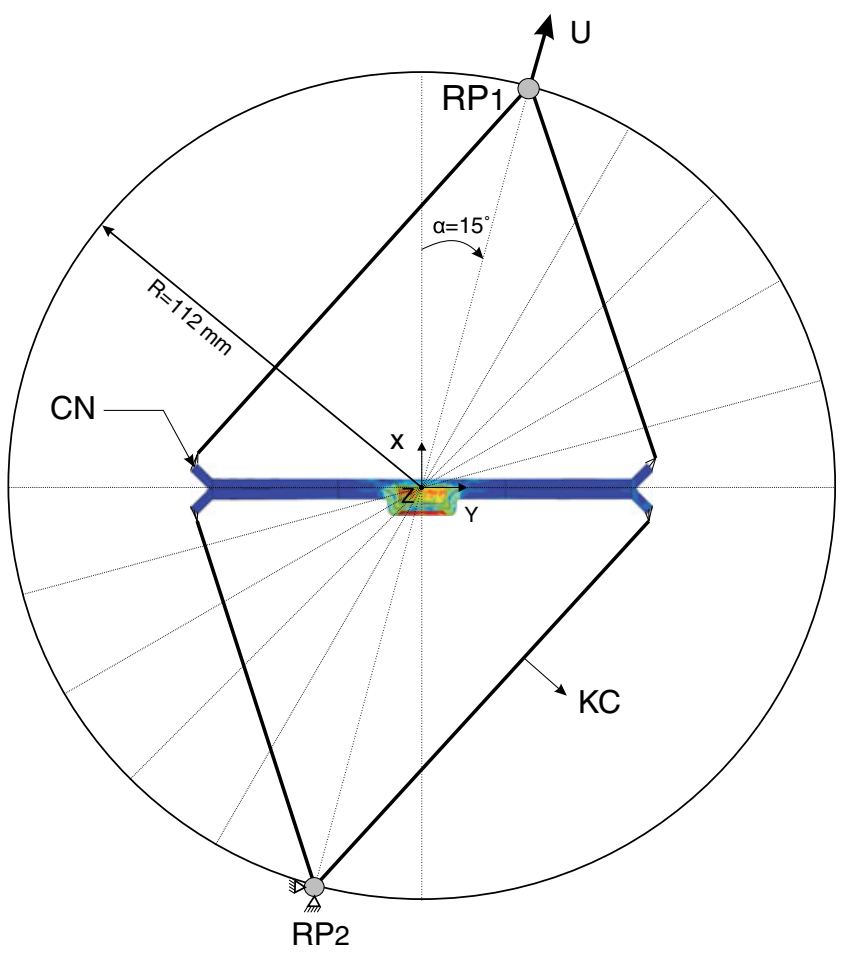

Fig. 12 Cross section along the plane of symmetry of the FE model. $K C$ kinematic coupling, $R P 1$ reference point $1, R P 2$ reference point 2, $U$ magnitude of the rigid body motion and $C N$ coupling nodes

directions while rotations can be transmitted around the local $X$ and $Z$ axis. Due to the $Z$-symmetry only half the Arcan specimen is modelled. All simulations in this section are carried out using the commercial FEA code Abaqus/Explicit. Models are meshed (15 elements through the thickness of the sheet) using 8-node linear bricks with reduced integration and hourglass control. The principal material directions in the models which take planar anisotropy into account are oriented with respect to the local coordinate system of the sheets shown in Fig. 12. The rolling direction (RD) in these simulations corresponds with the $Y$-direction, and, as a consequence, the transverse direction (TD) coincides with the $Z$-direction.

\section{Results and discussion}

The modified Arcan device is mounted in a tensile machine from Zwick Roell with a load capacity of $10 \mathrm{kN}$. All tests are conducted with a constant cross-head speed of $1 \mathrm{~mm} / \mathrm{min}$. The joint shown in Fig. 1 is tested under multi-axial loading conditions. For each angular position $\alpha$ three Arcan specimens are tested, and, as a result, a total of 21 tests are performed. Figure 13 shows the results of the first run for all angular positions.

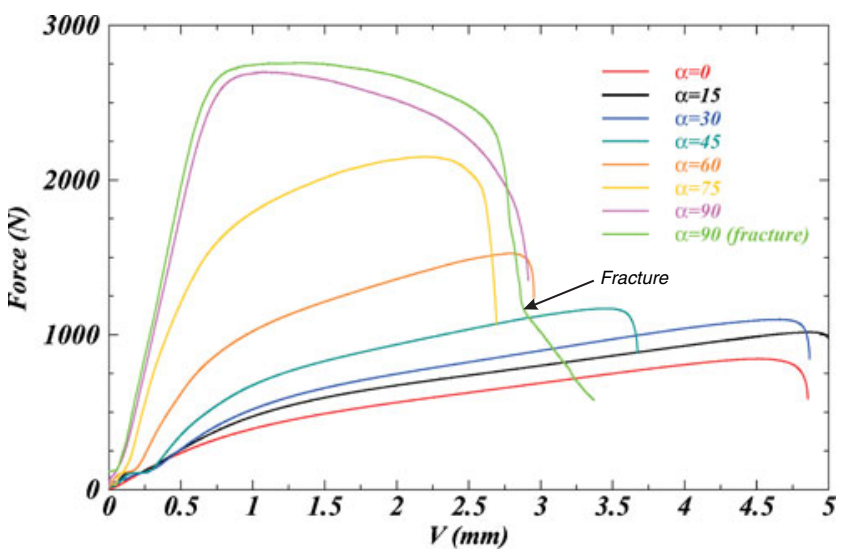

Fig. 13 Force displacement curves for the various angular positions

As expected, it can be inferred from this figure that the shear/tensile ratio severely influences the maximum strength and the ductility of the connection. In addition, the figure shows that the ratio has a significant influence on the joint's stiffness. If the contribution of the pull-out component is large, the surrounding sheet material is deformed rather than the joint itself. There is evidence to suggest that when shear loading starts to dominate, the plastic deformation is concentrated in the vicinity of the joint resulting in a much stiffer behaviour. Figure 14a and b show the dependency of the maximum force and the ductility with respect to the angular position $\alpha$, respectively. It is clear that increasing the shear component leads to a higher joint strength. From the Fig. 14a it can be inferred that the rate with which this occurs is much larger beyond the angular position $\alpha=45^{\circ}$.

It can also be inferred that the $\alpha=45^{\circ}$ loading case yields the lowest standard deviation. The same observation is reflected in the joint ductility, as can be seen from Fig. 14b. For both the maximum strength and the ductility, the $\alpha=45^{\circ}$ loading case yields the lowest standard deviations followed by the loading cases $\alpha=0^{\circ}$ and $\alpha=90^{\circ}$. For all other shear/tensile ratios the standard deviations are significantly larger. From Fig. 14b it can be derived that the ductility has two levels with $\alpha=45^{\circ}$ being in the threshold zone.

The numerical model described in the previous section is used to predict the joint strength under multiaxial loading. The purpose of this paper is also to investigate the quality of the identified material and frictional behaviour. Therefore the numerical model is "fed" with the data sets from Table 2. The identification of the post-necking hardening behaviour is obtained with three independent tests. The frictional behaviour, however, is identified through inverse modelling by 


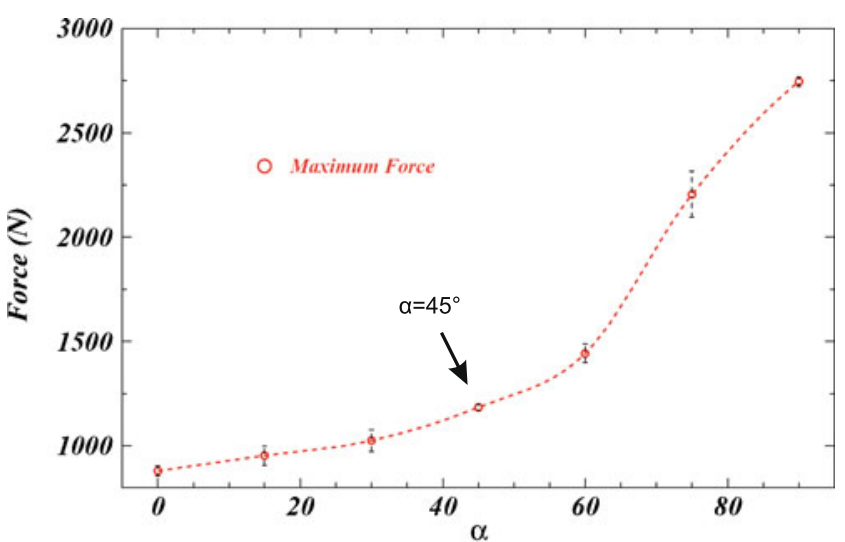

(a)

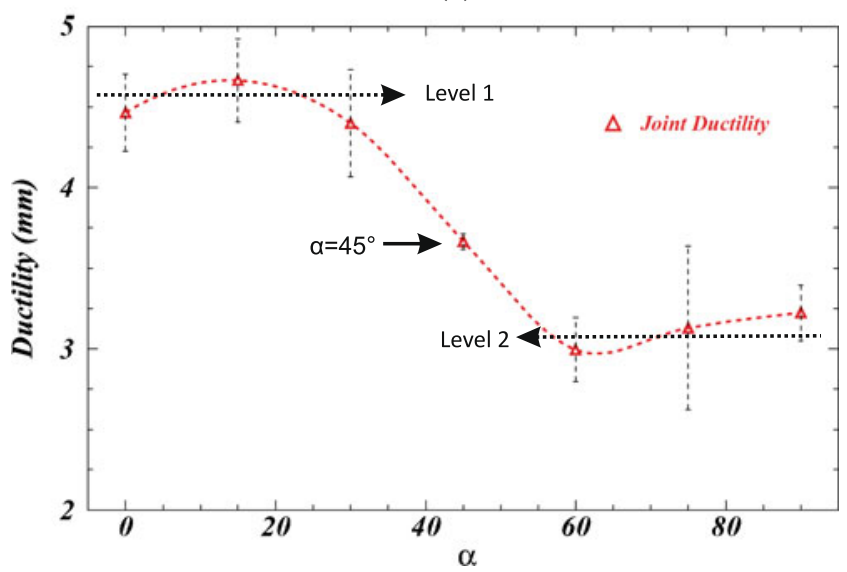

(b)

Fig. 14 a Peak loads. Right panel $\mathbf{b}$ ductility of the joint

taking into account the interlock $F$ and the process curve. As a consequence, all data sets from Table 2 yield approximately the same joint in terms of geometry and process graph.

Since for pull-out testing $\left(\alpha=0^{\circ}\right)$ the frictional dissipation is larger than the plastic deformation of the joint, this particular loading case will allow the assessment of the identified inter-sheet frictional behaviour $\left(\mu_{i}^{k}\right)$ and the interlock $F$. On the other hand, plastic dissipation predominates in shear loading which in turn allows the assessment of the identified material behaviour. Since the alternative material tests, EM and PNTE, make use of more experimental data to identify post-necking hardening behaviour, it can be expected that these tests yield more consistent results. In other words, the data sets should be less dependent on the hardening laws which are chosen a priori. Figures 15 and 16 show the results of the numerical models along with the experimental results for $\left(\alpha=0,15,30^{\circ}\right)$ and $\left(\alpha=45,60,75^{\circ}\right)$, respectively. Figure 17 shows the results for $\left(\alpha=90^{\circ}\right)$.

According to these figures, it appears that all optimized data sets from Table 2 yield similar results for

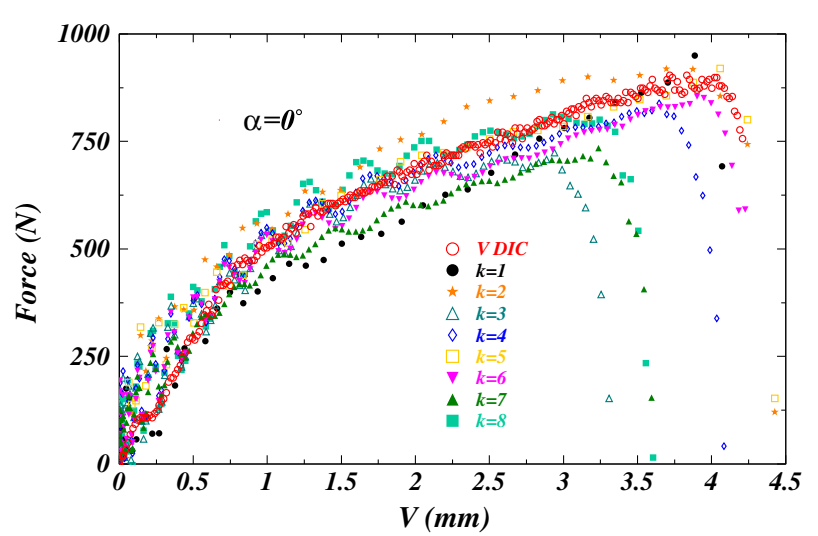

(a)

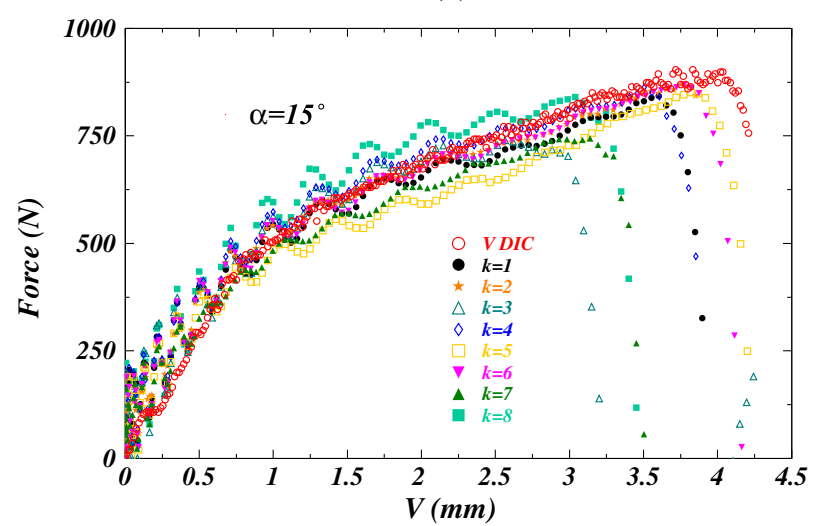

(b)

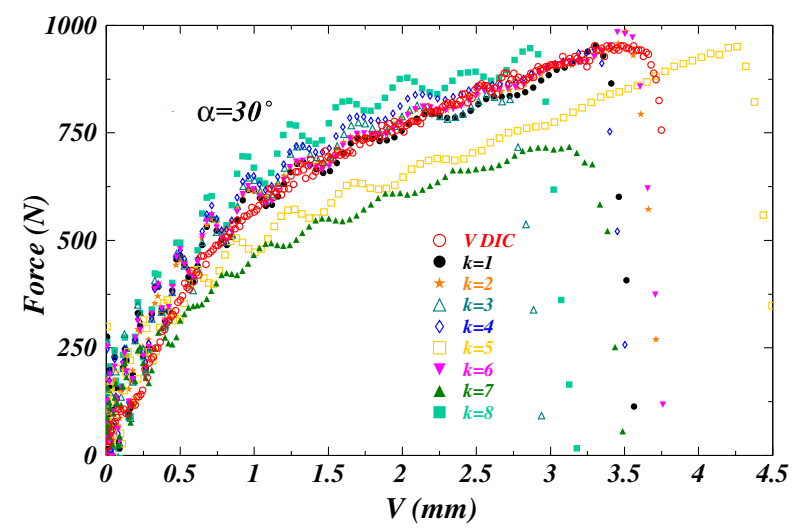

(c)

Fig. 15 Force-displacement graphs for the different angular positions $\left(\alpha=0,15,30^{\circ}\right)$

all multi-axial loading cases. The first two data sets $k=1,2$ yield in pull-out testing $\left(\alpha=0^{\circ}\right)$ different evolutions of the process graph, however, similar maximum strengths are predicted. Since the interface friction $\mu_{i}$ is identical in both simulations, the difference is caused by a slightly larger interlock owing to the use of the Voce hardening law. Accordingly, this causes the model to predict higher intermediate forces in pure pullout testing. This effect disappears with an increasing shear/tensile ratio. 


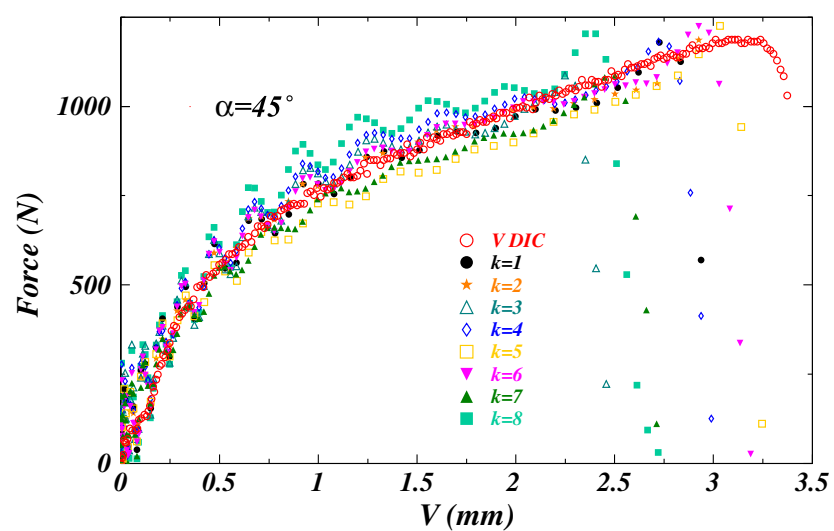

(a)

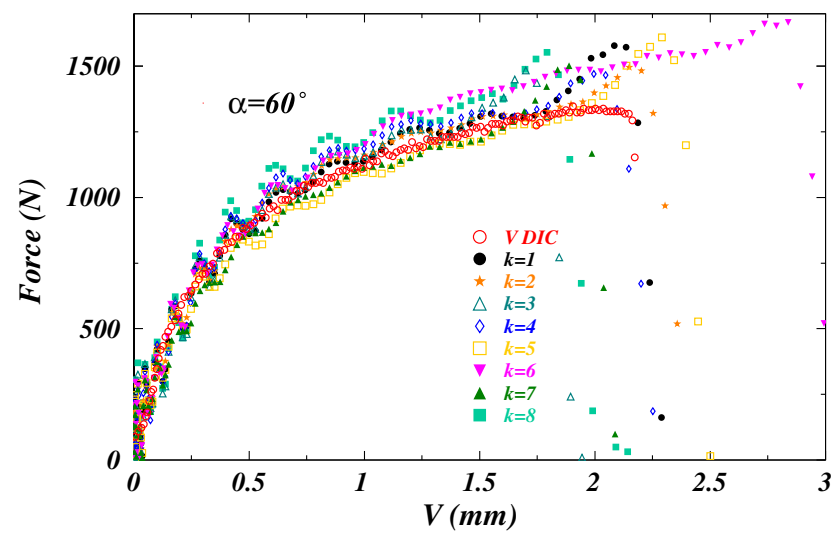

(b)

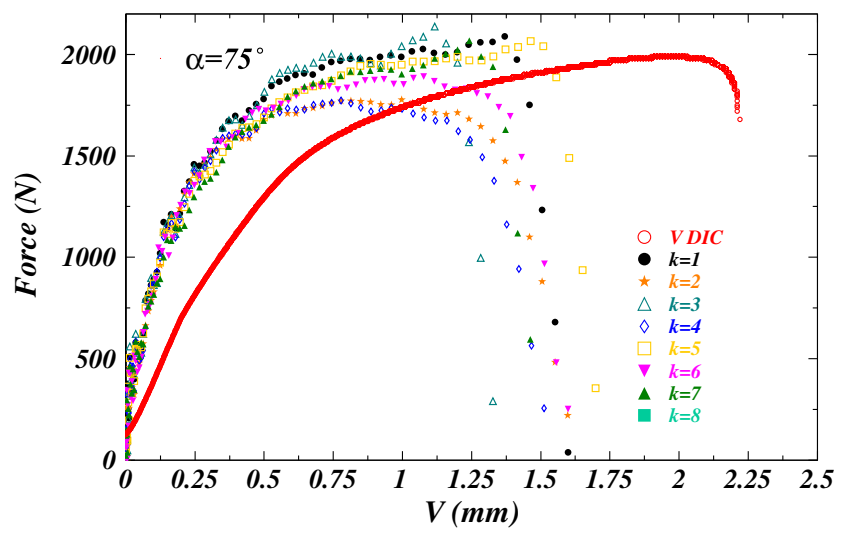

(c)

Fig. 16 Force-displacement graphs for the different angular positions $\left(45,60,75^{\circ}\right)$

The discrepancy reappears when $\alpha=75^{\circ}$ and is maximal for $\alpha=90^{\circ}$, however, the reason for this is different. For $\alpha=75^{\circ}$, the Swift model starts to predict a much higher maximum force than the Voce model. Moreover, in shear loading, the Swift model predicts forces which are closer to the experimental values. Clearly, the material model starts to play a significant role when the amount of plastic deformation increases

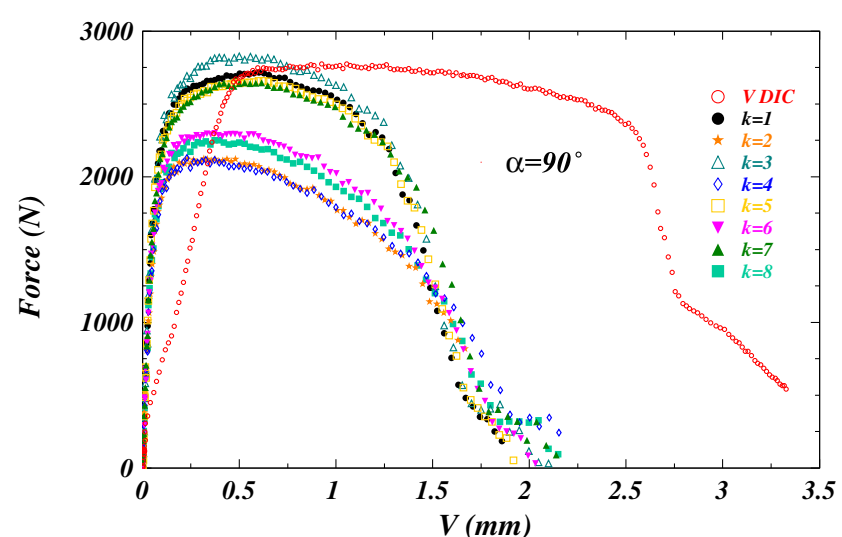

(a)

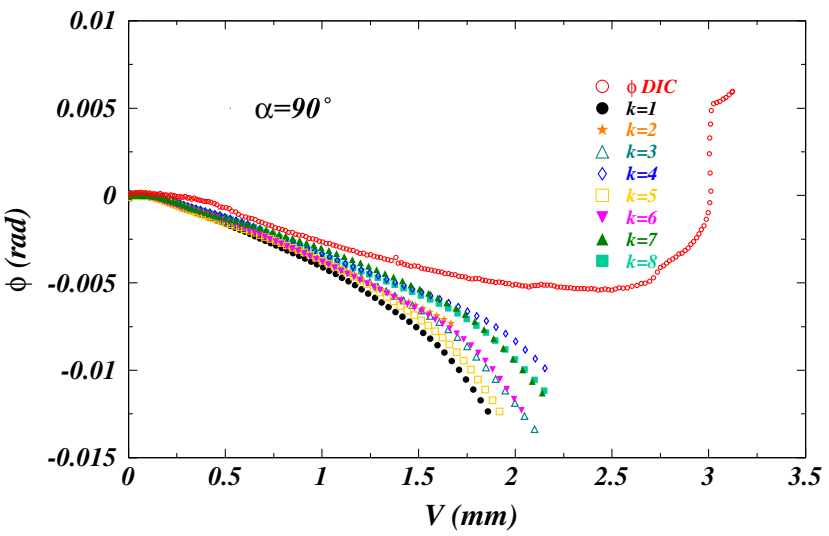

(b)

Fig. 17 Force-displacement graphs for the different angular positions $\left(45,60,75^{\circ}\right)$

and in this case, $\alpha=75^{\circ}$ introduces enough shear loading to reveal this behaviour.

Although identical trends apply for all identified data sets, there is some dissimilarity regarding the consistency of the results. As already stated, for $(\alpha=$ $\left.0,15,30,45,60^{\circ}\right)$ the optimized data sets $(k=1-8)$ yield very similar results. However, for the angular position $\alpha=75^{\circ}$ and $\alpha=90^{\circ}$ the alternative material data sets yield more consistent results in comparison with the EM in the sense that the differences between the adopted hardening law are smaller. Indeed, the difference remains quite large for $\alpha=90^{\circ}$, but the effort of identifying post-necking hardening behaviour is still clearly visible.

It can be concluded that the Swift hardening models identified through the different material tests yield the best results. This effect is more pronounced when substantial shear loading is present. As such, it can be stated that the prediction of the shear strength highly depends on the accuracy of the material model. In the case of pull-out loading all identified hardening models yield satisfactory results which suggests that the 
accuracy of final geometry is predominant in this type of simulation.

The orientation of the principal material directions in the models which take planar anisotropy into account are defined in Section "FE model". The influence of this orientation on the mechanical behaviour is investigated and Fig. 18 shows the results. These curves are obtained by simulation of the angular position $\alpha=90^{\circ}$ using data set $k=7$, see Table 2 . The curve labelled as $k=7 Y=R D ; Z=T D$ is obtained with the principal material directions oriented as described in Section "FE model". A second model in which the principal material directions are rotated by $90^{\circ}$ is built. The result from the latter model is labelled as $k=7$ $Y=T D ; Z=R D$ in Fig. 18. It can be inferred from this figure that the planar anisotropy has a marginal effect on the mechanical behaviour.

Furthermore, there are also some artefacts to be reported in the case of substantial shear loading. First, from Figs. 16c and 17a it appears that for $\alpha=75^{\circ}$ and $\alpha=90^{\circ}$ the stiffness of the joint is overestimated. It is found that this caused by an inaccurate measurement of the displacement V. Indeed, in shear loading the force increases very rapidly in the beginning of the test. This is accompanied with very small relative displacements between the sheets. Since the measurement of $\mathrm{V}$ is conducted on the rigid disks by DIC (or by the movement of the cross head far away from the joint), these small displacements cannot be accurately captured by the current system. This can only could be remedied by using an extensometer in the close vicinity of the joint. However, this requires the Arcan device to be adapted.

A second artefact is the poor prediction of the rotation of the disk half by the numerical models as shown in Fig. 17b. Clearly, all numerical models overestimate this rotation. This is a numerical error which originates

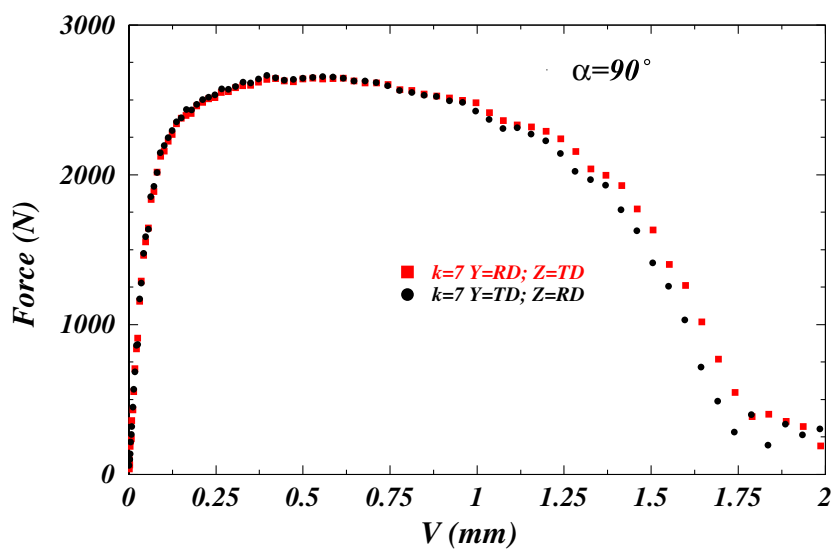

Fig. 18 Influence of the orientation of the principal material directions on the mechanical behaviour from the "perfect" boundary definition of pin 2, see Fig. 2. During the actual test, the rotation of a disk half is hampered by friction between this pin and the disk half. As discussed in Section "FE model", this pin is modelled by a reference point which allows the disks to rotate in the plane of the disks. The problem can be remedied by fixing the disk half as explained in Section "The modified Arcan setup". However, this might cause a harmful side load in the load cell.

Finally, a third artefact is that the numerical models ( $\left.\alpha=0,15,30,45,60^{\circ}\right)$ often predict a wobbling curve. At present, the reason for this is not fully understood and should be investigated in more detail.

Figure 19 shows the failure modes for $(\alpha=0$, $\left.15,30,45,60,75^{\circ}\right)$ and for all three replicas in each angular position $\alpha$ the same mechanism is observed: failure by deformation. For $\alpha=90^{\circ}$ two of the three specimens failed by deformation, one specimen developed a crack which propagated through the neck of the joint. The latter phenomenon is illustrated by Fig. 20 . The experimental results in Figs. 19 and 20 are accompanied by the numerical computed shapes just before the connection fails. It can be inferred from Fig. 19 that the shapes are fairly reproduced. The rotation of the die side is gradually increasing from $\alpha=75^{\circ}$ to $\alpha=90^{\circ}$ and there are no visible cracks. Also for $\alpha=90^{\circ}$ the overall deformation mode is reproduced quite accurately. It is worth noting that the die side does not rotate in the latter loading case.

Since the FE model does not incorporate a damage model, the fracture observed in the lower panel of Fig. 20 could not be reproduced. However, this fracture occurred in a very late stage of the shear test as indicated by the black arrow in Fig. 13, and, as a consequence, the model is still capable of predicting the maximum force. Another consequence of the absence

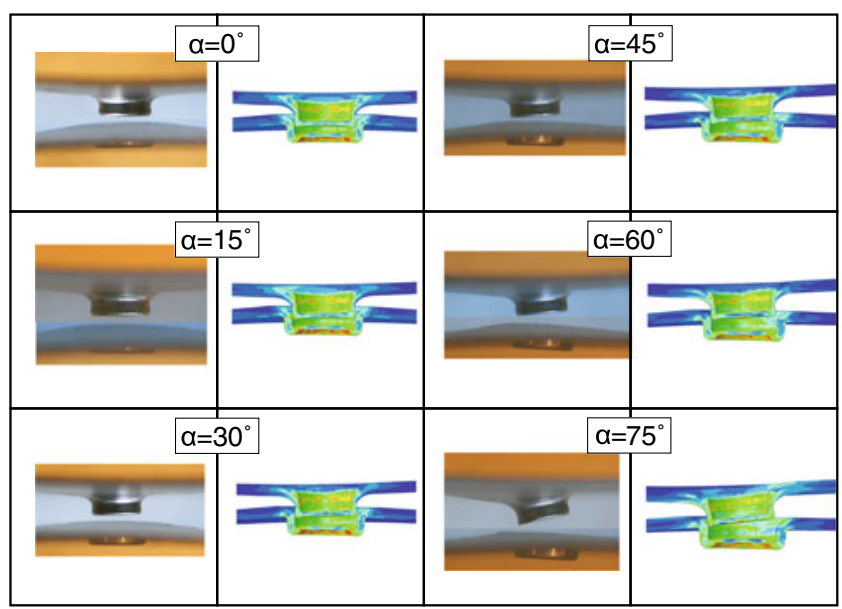

Fig. 19 Deformation modes for $\left(\alpha=0,15,30,45,60,75^{\circ}\right)$ 


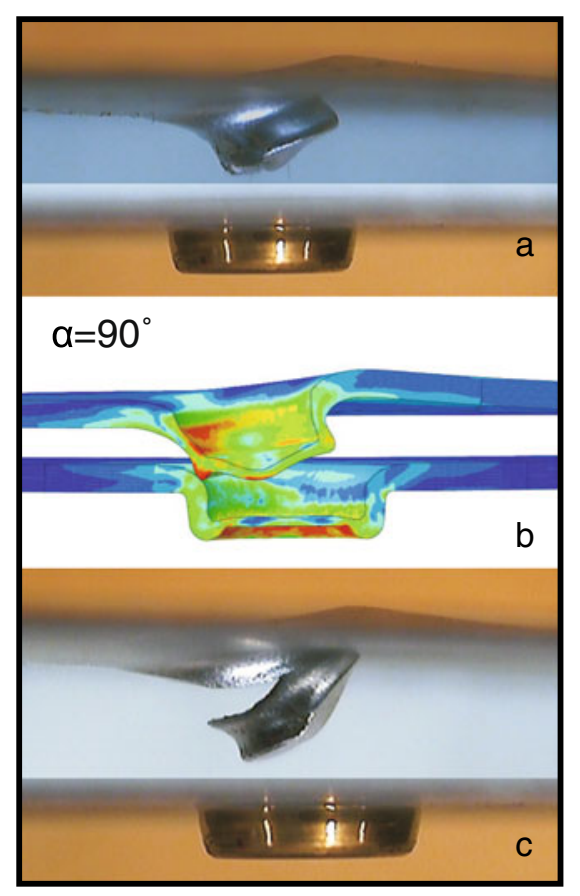

Fig. 20 Deformation modes of the joint under shear loading in the Arcan set up $(\alpha=90)$. a No fracture, b simulation, $\mathbf{c}$ ductile fracture

of a damage model is the large discrepancy in the prediction of the rotation of the disk halve shown in Fig. 17b. Indeed, the propagation of a crack influences the rotation of the Arcan fixtures. This is clearly illustrated in Fig. 17b: large deviations are observed beyond the maximum strength $(\mathrm{V} \approx 1 \mathrm{~mm})$.

\section{Conclusion and future work}

This contribution presents results on the mechanical behaviour of clinched connections under multi-axial loading conditions. The first part is concerned with the development of an experimental method which enables to exert various shear/tensile ratio's on clinched connections. An Arcan-like setup is designed and equipped with the digital image correlation technique to measure all rigid body motions of the fixtures. The second part embarks on the strategy to simulate the forming of a clinched joint which involves the identification of postnecking hardening behaviour of sheet metal and frictional behaviour. The post-necking hardening behaviour of DC05, a mild deep drawing steel, is identified through three methods. Since friction plays a vital role in clinch forming, the unknown friction coefficients are inversely identified based on the experimentally measured interlock and the process graph. In the third part, the multi-axial experiments on a DC05-DC05 clinched connection are used to validate FE models of the test with varying shear/tensile ratios.

It is shown that the maximum strength of the connection increases when the magnitude of the shear component increases, between $\alpha=45^{\circ}$ and $\alpha=90^{\circ}$ the strength increases more rapidly than between $\alpha=0^{\circ}$ and $\alpha=45^{\circ}$. The ductility of the joint appears to have two levels which are separated by a transition zone around $\alpha=45^{\circ}$. Increasing the magnitude of the pullout component leads to an increased joint ductility. Each material data set together with the identified frictional properties are used to simulate seven different shear/tensile ratios. In general, the presented FE model is able to reproduce the experimental observations such as the force-displacement curve and the deformation mode of the joint. Since the numerical model does not incorporate a damage model, fracture in the neck could not be reproduced. Unlike the conventional way to identify post-necking hardening laws of sheet metal, it is shown through the multi-axial tests on clinched joints that the alternative material tests yield a hardening behaviour which is less dependent on the hardening law which needs to be chosen a priori. The significance of this is illustrated when there is sufficient plastic deformation of the joint, and, as a consequence, when the shear component reaches a critical value.

Future work should embark on improving the measurement accuracy of the relative displacement between the sheets. It should also be investigated why the numerical models sometimes predict a wobbling curve.

\section{References}

1. Nong N, Ouyang K, Zhang Y, Qiao Z, Tao C, Li F (2003) Research on press joining technology for automotive metallic sheets. J Mater Process Technol 137:159-163

2. Neugebauer R, Kraus C, Dietrich S (2008) Advances in mechanical joining of magnesium. CIRP Ann Manuf Technol 57:283-286

3. Pirondi A, Moroni F (2011) Science of clinch-adhesive joints. Adv Struct Mater 6:109-147

4. Sadowski T, Balawender T (2011) Technology of clinchadhesive joints. Adv Struct Mater 6:149-176

5. Hamel V, Roelandt JM, Gacel JN, Schmit F (2000) Finite element modeling of clinch forming with automatic remeshing. Comput Struct 77:185-200

6. de Paula AA, Aguilar MTP, Pertence AEM, Cetlin PR (2007) Finite element simulations of the clinch joining of metallic sheets. J Mater Process Technol 182:352-357

7. Coppieters S, Cooreman S, Lava P, Sol H, Van Houtte P, Debruyne D (2010) Reproducing the experimental pull-out and shear strength of clinched sheet metal connections using FEA. Int J Mater Form. doi:10.1007/s12289-010-1023-6

8. Oudjene M, Ben-Ayed L, Delamézière A, Batoz J.-L. (2008) Shape optimization of clinching tools using the response 
surface methodology with moving least-square approximation. J Mater Process Technol 209:289-296

9. Saberi S, Enzinger N, Vallant R, Cerjak H, Hinterdorfer J, Rauch R (2008) Influence of plastic anisotropy on the mechanical behaviour of clinched joint of different coated thin steel. Int J Mater Form Suppl 1:273-276

10. Joma M, Billardon R (2007) Numerical analysis of the resistance to pullout test of clinched assemblies of thin metal sheets. In: Proceedings of the 10th ESAFORM conference, pp 169-174

11. Langrand B, Combescure A (2004) Non-linear and failure behaviour of spotwelds: a "global" finite element and experiments in pure and mixed modes I/II. Int J Solids Struct 41:6631-6646

12. Langrand B, Patronelli L, Deletombe E, Markiewicz E, Drazétic P (2002) An alternative numerical approach for full scale characterisation for riveted joint design. Aerosp Sci Technol 6:343-354

13. Arcan M, Hashin Z, Voloshin A (1978) A method to produce uniform plane-stress with applications to fiber-reinforced materials. Exp Mech 35:141-146

14. Sutton MA, Boone ML, Ma F, Helm JD (2000) A combined modeling-experimental study of the crack opening displacment fracture criterion for characterization of stable crack growth under mixed mode I/II loading in thin sheet metals. Eng Fract Mech 66:171-185

15. Kavamura HA, Batalha GF (2008) Mechanical strength evaluation for Nd-YAG laser and electric resistance spot weld (ERSW) joint under multi-axial loading. J Mater Process Technol 201:507-514

16. Patronelli L, Langrand B, Deletombe F, Markiewicz E, Drazétic P (1999) Analysis of riveted joint failure under mixed mode loading. Eur J Mech Environ Eng 44:223228

17. Porcaro R, Hanssen AG, Langeth M, Aalberg A (2006) The behaviour of a self-piercing riveted connection under quasi-static loading conditions. Int J Solids Struct 43:5110 5131
18. Greer JM, Galyon Dorman SE, Hammond MJ (2011) Some comments on the Arcan mixed-mode (I/II) test specimen. Eng Fract Mech 75:2088-2094

19. Merklein, M, Gödel, V (2009) Characterization of the flow behaviour of deep drawing steel grades in dependency of the stress state and its impact on FEA. Int J Mater Form Suppl 2:415-418

20. Maeda, Y, Yanagawa, M, Barlat, F, Chung, K, Hayashida, Y, Hattori, S, Matsui, K, Brem, JC, Lege, DJ, Murtha, SJ and Ishikawa, T (1998) Experimental analysis of aluminum yield surface for binary Al-Mg alloy sheet samples. Int J Plasticity 14(4-5):301-318

21. Kajberg, J, Lindkvist, G (2004) Characterisation of materials subjected to large strains by inverse modelling based on inplane displacement fields. Int J Solids Struct 41:3439-3459

22. Rossi, M, Pierron, F (2012) Identification of plastic constitutive parameters at large deformations from three dimensional displacement fields. Comput Mech 49:2492-2497

23. Coppieters S, Lava P, Sol H, Van Bael A, Van Houtte P, Debruyne D (2010) Determination of the flow stress and contact friction of sheet metal in a multi-layered upsetting test. J Mater Process Technol 210:1290-1296

24. Coppieters S, Cooreman S, Sol H, Van Houtte P, Debruyne D (2011) Identification of the post-necking hardening behaviour of sheet metal by comparison of the internal and external work in the necking zone. J Mater Process Technol 211: $545-552$

25. Totten GE, Funatani K, Xie L (2004) Handbook of metallurgical process design. Marcel Dekker, Inc. New York

26. Coppieters S, Lava P, Sol H, Van Houtte P, Debruyne D (2011) Identification of post-necking hardening behaviour of sheet metal: a practical application to clinch forming. Key Eng Mater 473:251-258

27. Joma M, (2007) Simulation numerique des procedes de clinchage et de rivetage pour application automobile. $\mathrm{PhD}$ thesis, Université Pierre et Marie Curie, France

28. Mucha J, (2011) The analysis of lock forming mechanism in the clinching joint. Mater Design 32:4949-4954 